\title{
POSSIBILITY OF A WHITE DWARF AS THE ACCRETING COMPACT STAR IN CI Cam (XTE J0421+560)
}

\author{
Manabu Ishida, ${ }^{1}$ Kazuyuki Morio, ${ }^{2}$ and Yoshihiro Ueda ${ }^{2}$ \\ RESUMEN
}

Aunque CI ('am, la cual presentó uná explosión en abril de 1998, ha sido considerada como una fuente transitoria de rayos X suaves (SXT) que alberga un agujero negro, posee a la vez ciertas características que difícilmente pueden hacerla encajar dentro del grupo de SXT. En este artículo proponemos una enana blanca como la estrella central acreciente en CI Cam.

\begin{abstract}
Although CI Cam, which showed an outburst in April 1998, has been regarded as a soft X-ray transient (SXT) harboring a black hole, it has some characteristics that can hardly be reconciled with the SXT picture. In this paper, we propose a white dwarf as the central accreting star in CI Cam.
\end{abstract}

Key Words: STARS: INDIVIDUAL (CI CAM, XTE J0421+560) - X-RAYS: STARS

\section{INTRODUCTION}

CI Cam was discovered in an outburst on 1998 March 31 (Snith et al. 1998) with the All-sky Monitor onboard Rossi $X$-ray Timing Explorer. Its X-ray flux reached the peak of $\sim 2$ Crab on April 1.04. On the basis of the updated distance to CI Cam of 5$9 \mathrm{kpc}$ (Robinson et al. 2002; Hynes et al. 2002), the burst peak luminosity in the $225 \mathrm{keV}$ band results in $L_{\mathrm{X}}=(3-10) \times 10^{38} \mathrm{erg} \mathrm{s}^{-1}$. A high ratio of this $\mathrm{X}-$ ray luminosity to that of optical leads Robinson et al. (2002) to conchude that CI Cam is a new soft X-ray transient (SXT) harboring a black hole (BH). The outburst of ('I Cam, however, showed several characteristics that have never been secn from any other BH SXT. First, the decay of X-ray flux from the peak is quick with an e-folding time of order a day, which is too short for the BH SXTs (several tens of days), and is accompanied with no short-term flickering (Belloni ct al. 1999). Sccond, the burst peak occurs later in a longer warelind (Frontera et al. 1998). This is reminiscent of ejected gas showing a transition from an optically thick to thin state due to expansion. The SXT outburst is, on the other hand, understood as an accretion crent. Finally, the hard X-ray spectra taken by $A S C A$ (Ueda et al. 1998) and Beppo-SAX (Orr et. al. 1998) within three days since the outburst onset showed the optically thin nature.

In this paper we will show some pieces of evidence indicating that the central object of CI Cam is a white dwarf (WD). Refer to Ishida ot al. (2004) in more detail of this proceeding paper.

\footnotetext{
${ }^{1}$ Tokyo Metropolitan Lniversity, Japan.

2ISAS/JAXA, Japan.
}

\section{OBSERVATIONS AND RESULTS}

\subsection{ASCA X-ray Spectrum in Quiescence}

In Fig. 1 shown are the $A S C A$ spectra of CI Cam taken during 1999 February 19-20. They are un-

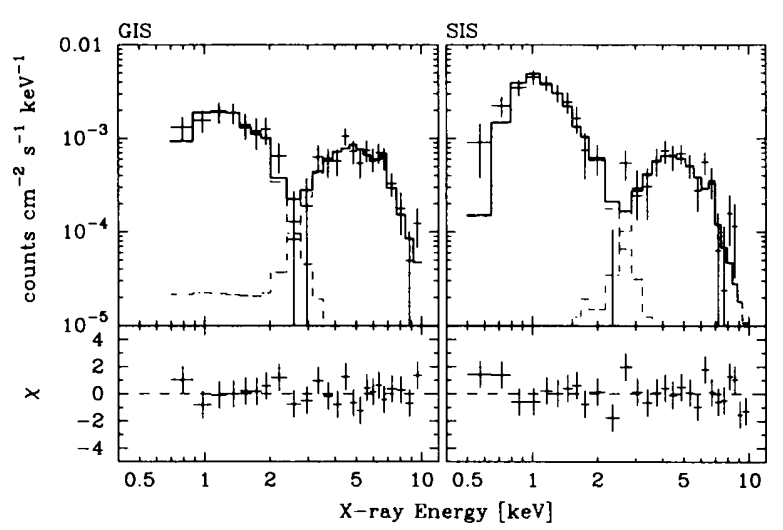

Fig. 1. ASCA spectra of CI Cam in quiescence. Left and right panels are from the GIS and the SIS, respectively.

ambiguously composed of two components separated at around $2-3 \mathrm{keV}$. Of them, the hard component, which originates from the accreting compact object, is most naturally interpreted as the optically thin thermal plasma emission owing to a strong iron $\mathrm{K} \alpha$ emission line. This is a characteristic of cataclysmic variables, accreting WD binaries.

\subsection{ASCA $X$-ray Data in Outburst}

The ASCA SIS light curve on 1998 April 3 in the $0.5-1.0 \mathrm{keV}$ band is shown in Fig. 2. This soft band light curve is characterized by sudden appearance of a soft component independent of the underlying hard component which decays exponentially. 


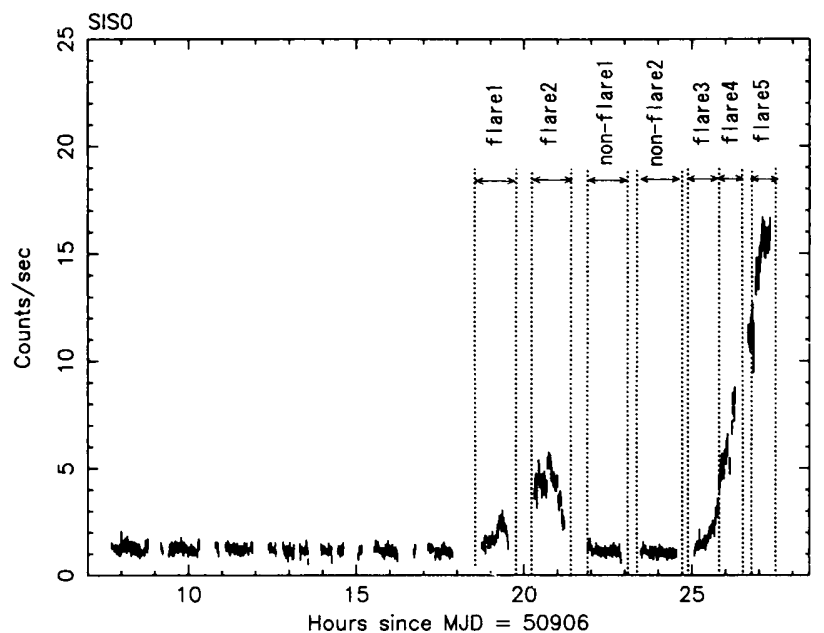

Fig. 2. $A S C A$ SISO light curve in the $0.5-1.0 \mathrm{keV}$ batid in outburst.

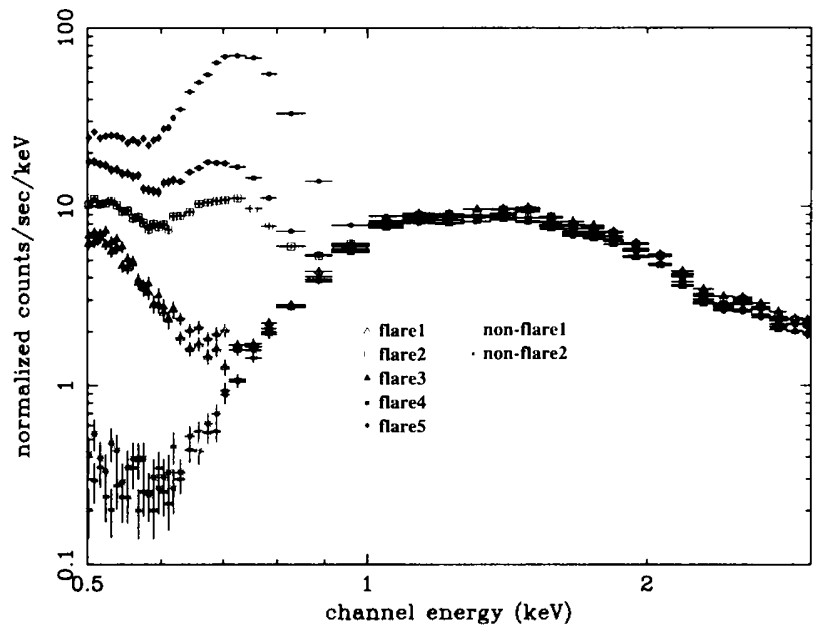

Fig. 3. ASCA SIS spectra of CI Cam in the five flare phases delineated in Fig. 2.

The spectra of the seven data segments (flare 1-5 and non-flare 1-2) up to $3 \mathrm{keV}$ are shown in Fig. 3. From spectral fitting, it is found that the soft components are characterized by a black body overlaid with hydrogenic and He-like Oxygen K-edges. The temperatures vary within the range $0.07-0.12 \mathrm{keV}$, and the peak (flare 5 phase) bolometric luminosity is consistent with $10^{38} \mathrm{erg} \mathrm{s}^{-1}$. All of these characteristics are common with super-soft sources (SSSs) that host a WD as the central accreting object, for example, CAL 87 (Asai et al. 1998; Ebisawa et al. 2001).

\subsection{Distance Estimation from Optical Light curve}

Since a nova, whose outburst is a thermo-nuclear flash on the surface of an accreting WD, has been known to show the properties of a SSS in the declining phase (Krautter et al. 1996; Starrfield et al. 2000; Orio et al. 2002), it seems possible to interpret the outburst of CI Cam as a nova outburst. It is well known that there is a tight correlation between the burst peak absolute magnitude and the burst decay time (c.g. Cohen (1988)). The nova hypothesis can thus be tested by cstimating the distance based on an optical decaying light curve, which we took from VSNET database. As a result, the distance to CI Cam is obtained to be $4.517 .4 \mathrm{kpc}$, which is consistent with the updated distance cistimate of 5, $9 \mathrm{kpc}$.

\section{SUMMARY}

The hard $\mathrm{X}$-ray emission albow $2 \mathrm{keV}$ in quiescence is likely to bo an optically thin thermal plasma emission, which is a characteristic of catidclysmic variables, accreting WD binaries. The outburst spectrum within thre days from the onset shows a soft X-ray component which resembles that of SSSs. Since a nova is known to show the characteristics of SSSs in the cleclining phase, we have assumed a nova outburst for CI ('an and have stimated the distance by referring 10 the optical decay light curve. The resulting distance is consistent with that updated with other method. All these mints indicate that the central object of CI Cam is a WD.

\section{REFERENCES}

Asai, K., Dotani, T., Hoshi, R., Tanaka, Y., Robinson, C. R., \& Terada, K. 1998b, PASJ, 50, 611

Belloni, T., et al. 1999, ApJ, 527, 3.15

Cohen, J. G. 1988, in ASP Conf. S'r. 1. The Extragalactic Distance Scalc, ed. S. van den Bergh \& ( J. Pritchet (San Francisco: ASP), 114

Ebisawa, K., Mukai, K., Kotani, T., Asai, K., Dotani, T., Nagase, F., Hartmann, H. W.. II'isc. J., Kahabka, P., \& van Teeseling, A. 2001, /apj, 5,50, 1007

Frontera, A. et al. 1998, A\&A, 339, L69

Hynes, R. I., et al. 2002, A\& $A, 392,991$

Ishida, M., Morio, K., \& Ueda, Y. 2004, ApJ, 601, in press

Krautter, J., Ögelman, H., Starrfield, S., Wichmann. W., \& Pfeffermann, E. 1996, ApJ, 456, 788

Orio, M., Parmar, A. N., Greiner, J., Ögelman, H., Starrfield, S., \& Trussoni, E. 2002, MNRAS, 333, L11

Orr, A., Parmar, A. N., Orlandini, M., Frontera, A., Dal Fiume, D., Segreto, A., Santangelo, A., \& Tavani, M. 1998, A\&A, 340, L19

Robinson, E. L., Ivans, I. I.. \& Welsh, W'. F. 2002. ApJ, 565. 1169

Smith, D., Remillard, R., Swank, J., Takeshima, T.. \& Smith, E. 1998, IAU: ('irc.. 68.55

Starrfield, S., et al. 2000, BAAS, 32. 4103

Ueda, Y., Ishida, M., Inoue, H., Dotani, T., Greiner, J., \& Lewin, W. H. G. 1998, ApJ, 508, L167 